Hendrik Neubauer und Jan Hemming (Kassel)

\title{
Wahrnehmung und Wirkung von Weihnachtsmusik im öffentlichen Raum
}

\section{Hintergrund und Fragestellung}

In der Weihnachtszeit ist im öffentlichen Raum überall Weihnachtsmusik zu hören. Doch wie nehmen die Menschen diese Musik wahr und welche Wirkung löst sie aus? Die hier vorliegende Untersuchung nimmt sich diesen Fragen an, auch weil sie bisher kaum erforscht wurden. Dabei stehen die Besucherinnen und Besucher des Märchenweihnachtsmarktes in Kassel und Menschen, die dort arbeiten, im Fokus.

\subsection{Akustische Räume des Märchenweihnachtsmarktes in Kassel}

Als städtischer Handlungsraum erstreckt sich der Märchenweihnachtsmarkt über drei Plätze (Königsplatz, Friedrichsplatz, Opernplatz), die sich an der Oberen Königsstraße, der Haupteinkaufsstraße der Kasseler Innenstadt, befinden. Für das Forschungsvorhaben hatte die Kassel Marketing GmbH, die den Weihnachtsmarkt im Auftrag der Stadt Kassel organisiert, freundlicherweise die genauen Lagepläne zur Verfügung gestellt. Ihnen ist zu entnehmen, dass jeder dieser Plätze aus Sub-Plätzen besteht. In Abhängigkeit von der Zeit hat jeder Sub-Platz seinen eigenen akustischen Raum, der mit Murray Schafer als der „Bereich, den ein Laut innerhalb einer Landschaft ausfüllt [...]"1, verstanden werden kann.

Für die Zeit des Märchenweihnachtsmarktes werden außen an der Einkaufsgalerie City Point Kassel, die sich an dem Sub-Platz „Rapunzelturmweg“ des Königsplatzes befindet, Lautsprecher angebracht. Am Friedrichsplatz und Opernplatz werden Lautsprecher an Gerüsten befestigt. Diese Installationen werden jährlich von der Kassel Marketing GmbH in Auftrag gegeben, um eine zentrale Beschallung mit Weihnachtsmusik zu gewährleisten. Zusammen mit anderen Klangquellen entsteht die akustische Umwelt bzw. Soundscape, ${ }^{2}$ für die sich an jedem Sub-Platz je nach Hörbezug ein eigener akustischer Raum ergibt. Liegt der Hörfokus auf der tonträgerwiedergegebenen Weihnachtsmusik, so lässt sich die Textur der akustischen Umgebung als $\mathrm{Hi}_{-} \mathrm{Fi}^{3}$ oder $\mathrm{Lo}-\mathrm{Fi}^{4}$ beschreiben. Hi-Fi bedeutet hier, dass die Weihnachtsmusik deutlich zu hören ist, während bei Lo-Fi verschiedene Signale überlagert werden, so dass die Musik nur undeutlich wahrzunehmen ist. Hierzu zählen z. B. Umgebungsgeräusche oder menschliche Stimmen. So kommt es zu dem Phänomen der Verdeckung bzw. Maskierung: „Ein Schall, der bei Ruhe noch gut gehört werden kann, wird so durch die Anwesenheit eines Störschalls unhörbar gemacht."

1 Raymond Murray Schafer, Die Ordnung der Klänge. Eine Kulturgeschichte des Hörens, dt. Übs. von Sabine Breitsameter, Mainz 2010, S. 433.

2 Ebd., S. 439.

3 Ebd., S. 434.

4 Ebd., S. 437.

5 Jobst P. Fricke und Christoph Louven, „Psychoakustische Grundlagen des Musikhörens“, in: Musikpsychologie. Das neue Handbuch, hrsg. von Herbert Bruhn, Reinhard Kopiez und Andreas C. Lehmann, Reinbek bei Hamburg ${ }^{3} 2011$, S. 416. 
Der akustische Raum ist nicht nur Teil der Soundscape, sondern, auf die wahrnehmenden Subjekte bezogen, auch Atmosphäre: „Die Atmosphäre ist die gemeinsame Wirklichkeit des Wahrnehmenden und des Wahrgenommenen. Sie ist die Wirklichkeit des Wahrgenommenen als Sphäre seiner Anwesenheit und die Wirklichkeit des Wahrnehmenden, insofern er, die Atmosphäre spürend, in bestimmter Weise leiblich anwesend ist." 6 Produzenten stellen Atmosphären her, Rezipienten erfahren die Präsenz von Menschen, Dingen und Umgebungen. ${ }^{7}$ Als Rezipienten treten vor allen Dingen sowohl die Besucherinnen und Besucher des Weihnachtsmarktes als auch die auf dem Weihnachtsmarkt tätigen Arbeitnehmerinnen und Arbeitnehmer auf. Beide Gruppen tauchen auf ihre Weise in das Klanggeschehen ein.

\subsection{Besucherinnen und Besucher des Weihnachtsmarktes}

Demnach könnte beispielsweise die Frage gestellt werden, inwieweit sich die Besucherinnen und Besucher des Märchenweihnachtsmarktes durch funktionell agierende Musik in ihrem Verhalten konditionieren und zum Konsum verführen lassen. Zunächst stehen für die Besucherinnen und Besucher verschiedene Erlebnisangebote zur Verfügung, deren akustische Räume u. a. mit Musik angereichert werden. Dabei sind nicht-kommerzielle und kommerzielle Angebote zu unterscheiden. Zu den nicht-kommerziellen Angeboten zählen Eröffnungsdarbietungen und Open-Air-Konzerte. Kommerzielle Angebote betreffen den Verkauf von Speisen / Getränken, verschiedenen Produkten (Lebensmittel, Kunsthandwerk, Bekleidung etc.) und Karten für Fahrgeschäfte. Außerdem sind die Besucherinnen und Besucher in selbst organisierte Gespräche involviert, die sich im Rahmen von nichtkommerziellen und kommerziellen Angeboten ergeben. Dabei können nicht-kommerzielle und kommerzielle Angebote gegenseitig sowie jeweils nicht-kommerzielle und kommerzielle Angebote untereinander von Nutzen sein: Beispielsweise können während eines OpenAir-Konzerts Speisen und Getränke verzehrt werden.

Publizierte Studien, die sich mit dem Einfluss von Musik auf Menschen in kommerzorientierten Verkaufs- und Gastronomieräumen beschäftigten, konzentrierten sich vor allem auf die Kundenwirkung und nähern sich teilweise der Beantwortung der o. g. Frage an. ${ }^{8}$ Günther Rötter und Catrin Plößner fanden in einer empirischen Feldstudie heraus, dass Hintergrundmusik in einem Supermarkt keinen Einfluss auf die Stimmung der Kunden hat und dass sie weder eine größere Aufenthaltsdauer noch eine Umsatzsteigerung bewirkt. ${ }^{9}$ Wird in einer Untersuchung die Art der Musik berücksichtigt, kann ein differenzierteres Ergebnis erwartet werden. Das Ergebnis einer Studie von Adrian C. North und David J. Hargreaves z. B. besagt, dass die Atmosphäre in einer Cafeteria positiv bewertet wird, wenn die dort wiedergegebene Musik mit der persönlichen Musikpräferenz korreli-

6 Gernot Böhme, Atmosphäre. Essays zur neuen Ästhetik, Frankfurt am Main 2013, S. 34.

7 Ebd., S. 25.

8 Z. B. Günther Rötter und Catrin Plößner, „Über die Wirkung von Kaufhausmusik“, in: Musikpsychologie, Empirische Forschungen - Ästhetische Experimente, hrsg. von Klaus-Ernst Behne, Günter Kleinen und Helga de la Motte-Haber, Wilhelmshaven 1994, S. 154-164; Adrian C. North und David J. Hargreaves, „The Effects of Music on Responses to a Dining Area“, in: Journal of Environmental Psychology 16.1 (1996), S. 55-64; Adrian C. North, David J. Hargreaves und Jennifer McKendrick, „In-store music affects product choice“, in: Nature 390 (13.11.1997), S. 132.

9 Rötter / Plößner, „Über die Wirkung von Kaufhausmusik“, S. 163. 
ert, was zum Wiederkommen einlädt. ${ }^{10}$ An dieser Stelle habe insbesondere anspruchsvolle Entspannungsmusik einen relevanten Einfluss auf die Kundenpflege. ${ }^{11}$ Später haben North und seine Mitarbeiter das Konzept des musical „fit“ aufgegriffen, das davon ausgeht, dass im Verkaufsraum eine speziell auf das Produkt abgestimmte Musik gespielt werden soll, um den Umsatz zu steigern. ${ }^{12}$ Diesen Effekt zeigten sie anhand verschiedener regionaler Weinprodukte, die mit landesspezifischer Musik in einen Zusammenhang gebracht wurden. Ebenso kann das Konzept des musical „fit“ für den Kontext des Weihnachtsgeschäfts ${ }^{13}$ bzw. des Weihnachtsmarktes nutzbar gemacht werden: Bei den kommerziellen Angeboten handelt es sich in der Regel um weihnachtliche Speisen / Getränke und weihnachtliches Kunsthandwerk, die in ihrem akustischen Raum von Weihnachtsmusik umgeben sind.

\subsection{Auf dem Weihnachtsmarkt tätige Arbeitnehmerinnen und Arbeitnehmer}

Ein weiterer Aspekt betrifft den psychosozialen Einfluss von Musik auf die Gruppe der auf dem Weihnachtsmarkt tätigen Arbeitnehmerinnen und Arbeitnehmer. Dabei kann Musik am Arbeitsplatz positive oder negative Auswirkungen haben. ${ }^{14}$ Auf der einen Seite können sich positive Einflüsse auf die Aktivierung und Motivation der Beschäftigten beziehen. Die andere Seite betrifft die negativen Einflüsse: Während Arbeitnehmerinnen und Arbeitnehmer an lärmintensiven Arbeitsplätzen, an denen Maschinen zum Einsatz kommen (z. B. in Fabrik- und Produktionshallen), zum Teil durch Schallschutzmaßnahmen entlastet werden können, ist eine Beschallung durch Musik im Handel bewusst und konzeptionell vorgesehen, ohne dass auf die akustischen Arbeitsbedingungen der Beschäftigten Rücksicht genommen wird. Besonders in saisonalen Spitzen wie der Vorweihnachtszeit wird in den Verkaufs- und Serviceräumen Musik eingesetzt, um die Kunden in Konsumlaune zu versetzen. Verkäufer/innen, Kassierer/innen, Köche und Köchinnen, gastronomische Servicekräfte sowie Reinigungskräfte werden gleichermaßen von der Musikbeschallung erfasst und während ihrer regelmäßigen Tätigkeit in den Verkaufs- und Serviceräumen ggf. belastet. Bei monotonen Tätigkeiten besteht aber auch die Möglichkeit, dass Musik entlastend wirkt.

Der akustische Raum, in dem sich die auf dem Weihnachtsmarkt tätigen Arbeitnehmer/innen befinden, wird nicht nur von der (mittelbaren) akustischen Umwelt beeinflusst. Seine Qualität und Intensität hängen maßgeblich von der (unmittelbaren) Umgebung ab, in der Musik in der zugehörigen Geschäftsstelle (z. B. Imbissstand, Getränkestand, Lebensmittelstand, Fahrgeschäftshäuschen etc.) wiedergegeben wird. Die hier eingesetzte Musik ist von ihrer Funktion her mit der Musik in Kaufhäusern vergleichbar. Die in der Regel leise Musik wird von Helmut Rösing als „akustische[...]r Teppich“ bezeichnet; bezweckt

10 North / Hargreaves, „The Effects of Music on Responses to a Dining Area“, S. 55-64.

11 Ebd.

12 North et al., „In-store music affects product choice“, S. 132.

13 Zein Kallas, Cristina Escobar und José M. Gil, „Assessing the impact of a Christmas advertisement campaign on Catalan wine preference using Choice Experiments", in: Appetite 58.1 (2012), S. 285291; Eric R. Spangenberg, Bianca Grohmann und David E. Sprott, „It's beginning to smell (and sound) a lot like Christmas: the interactive effects of ambient scent and music in a retail setting", in: Journal of Business Research 58.11 (2005), S. 1583-89.

14 Susanne Kunz, Musik am Arbeitsplatz, Wien 1991. 
würde „[...] eine allgemeine Verbesserung der Atmosphäre, eine Umsatzsteigerung [...] und eine Optimierung der Kommunikation zwischen Personal und Kunden." 15

\subsection{Weihnachtsmusik als Funktionelle Musik}

In der Regel handelt es sich bei der Musik auf dem Weihnachtsmarkt um Funktionelle Musik, die in den Hintergrund rückt. Bezogen auf Räume, in denen sich Arbeitnehmerinnen und Arbeitnehmer sowie Kunden aufhalten, fasst Rösing das Hauptziel des Einsatzes von Funktioneller Musik in „[...] die Steigerung von Leistung, Produktion und Umsatz [...], das heißt die Maximierung von Arbeitskraft und die Verführung zum Konsum [zusammen (H.N. und J.H.)]. Zu diesem Zweck soll Funktionelle Musik im Hintergrund [...] Atmosphäre schaffen, auch dort, wo eigentlich keine ist (Musik als akustisches Ornament mit Gestaltungsfunktion); [...] störende (Arbeits-)Geräusche übertönen (Musik zur Lärmabsorption); die Abgespannten aktivieren und die Gestreßten beruhigen (Musik zur Verhaltenskonditionierung); Nachdenken (z. B. kognitive Kaufblockade) verhindern durch das Verbreiten eines emotional positiven Bezugsrahmens (affektive Funktion von Musik)."16 Wie in vielen anderen öffentlichen Räumen ist auch bei der Musik auf dem Weihnachtsmarkt zu berücksichtigen, dass es sich hierbei aus der Sicht der Besucherinnen und Besucher um eine fremdbestimmte und seitens der Arbeitnehmerinnen und Arbeitnehmer um eine teilweise fremdbestimmte Musikauswahl (vgl. Kapitel 3.2.) handelt. Die Musikauswahl für die zentrale Beschallung treffen Dritte (vgl. Kapitel 2.1.2.), die dabei ein oder mehrere Motive im Sinn haben.

\subsection{Ziel der Untersuchung}

In der vorliegenden Untersuchung werden beide der beschriebenen Personengruppen berücksichtigt. Bei den Besucherinnen und Besuchern liegt der Fokus insbesondere auf der Wirkung und Nachwirkung der wahrgenommenen Weihnachtsmusik. Für letzteres bietet sich etwa die Frage nach eingetretenen Weihnachts-„Ohrwürmern“17 an. Grundsätzlich wird der Weihnachtsmarktbesuch als auditives Kulturerlebnis verstanden. Der psychosoziale Einfluss von Musik auf die Arbeitnehmerinnen und Arbeitnehmer kann dagegen Aufschluss darüber geben, welche Musik in welcher Arbeitszeitsequenz für welche Beschäftigungsgruppe positiv oder negativ bzw. entlastend oder belastend wirkt. Das Ergebnis dieser Untersuchung dient zunächst einer offenen Diskussion und könnte in eine Empfehlung münden, die Aspekte einer kundenorientierten Musikgestaltung beinhaltet, welche gleichzeitig arbeitnehmerfreundlich ist.

\section{Methoden und Durchführung}

Der Erhebungszeitraum erstreckte sich vom 25. November bis 25. Dezember 2014. Die gesamte Untersuchung wurde als Forschungsseminar an der Universität Kassel mit ca. 25 Teil-

15 Helmut Rösing, „Musikgebrauch im täglichen Leben“, in: Musikwissenschaft. Ein Grundkurs, hrsg. von Herbert Bruhn und Helmut Rösing, Reinbek bei Hamburg 1998, S. 118.

16 Ebd., S. 115.

17 Jan Hemming, „Zur Phänomenologie des,Ohrwurms “, in: Musikpsychologie - Musikalisches Gedächtnis und musikalisches Lernen, hrsg. von Wolfgang Auhagen u. a., Göttingen 2009, S. 184-207. 
nehmerinnen und Teilnehmern durchgeführt. Die Datenerhebung umfasste die Aufnahme von Soundscapes (1), ein Experteninterview (2), eine Erhebung unter Weihnachtsmarktbesucherinnen und -besuchern mit Kurzfragebögen (3) sowie themenzentrierte Interviews mit auf dem Weihnachtsmarkt tätigen Personen (4). Der Aufnahme von Soundscapes ging ein Pretest voraus, bei dem verschiedene Aufnahmeeinstellungen probiert wurden, um für die eigentliche Datenerhebung die für die jeweilige Situation optimale Einstellung zu finden. Ebenso wurde eine erste Version des Kurzfragebogens erprobt, so dass dieser vor seiner eigentlichen Anwendung optimiert werden konnte.

\subsection{Soundscapes und Experteninterview}

Der erste Teil der Erhebung bestand in der Aufzeichnung von Soundscapes und der Erstellung von Hörpartituren. Diese sollten es ermöglichen, auch im Nachhinein den akustischen Eindruck des Weihnachtsmarktes zu vergegenwärtigen. Die Soundscapes wurden an unterschiedlichen Orten und zu unterschiedlichen Zeitpunkten mit Hilfe von mobilen Audiorekordern aufgezeichnet. Die Notationen der Hörpartituren folgen zum Teil der Klassifizierung nach physikalischen Kriterien, so wie es Schafer ${ }^{18}$ beschreibt. An dieser Stelle werden die Parameter Dauer, Frequenz und Lautstärke abgebildet. Die Dauer verläuft an der x-Achse, Geräusche werden als Fläche aufgezeichnet (siehe unten), und die Lautstärke bildet sich als gleichmäßiger (Linie) oder schwankender Verlauf (Welle) in ihrer jeweiligen Intensität ab (siehe Abbildungen 1 und 2). Anhand von zwei exemplarisch ausgewählten Aufzeichnungen werden im Folgenden die Soundscapes beschrieben:

\subsubsection{Rapunzelturmweg (25. November 2014, $15.15 \mathrm{Uhr}$ )}

Die während der Aufnahme angefertigte Hörpartitur am Sub-Platz „Rapunzelturmweg“ vom 25. November 2014, 15.15 Uhr, umfasst die vier Klangschichten „Musikbox“, „Tram“, „Menschengemurmel“ und „Grill“. Mit „Musikbox“ ist die musikalische Beschallung des Weihnachtsmarktes gemeint, mit „Tram“ die Straßenbahn und mit „Menschengemurmel“ alles, was von Menschen zu hören ist. Der „Grill“ ist Teil eines Marktstandes direkt am Sub-Platz. Zu keiner Zeit tritt die Musik in der Soundscape besonders hervor, teilweise ist sie gegenüber anderen dominanten Geräuschen sogar kaum noch zu vernehmen. Bereits beim ersten Hören der Aufzeichnung wurde deutlich, dass die Spuren gegenüber der ursprünglichen Partitur noch sehr viel weiter zu differenzieren sind. Daraufhin wurden für die Neuanfertigung (siehe Abbildung 1) „Tram“ und „Grill“ übernommen, „Musikbox“ wurde allgemeiner als „Musik“ bezeichnet, und das „Menschengemurmel“ wurde in „Menschen“ und „Kinder“ aufgeteilt. Des Weiteren wurden die Spuren „Schritte“ und „Geldgeklimper“ hinzugefügt, da sie auf der Aufnahme recht markant erschienen. Gegenüber der ursprünglichen Hörpartitur erscheint die Neuanfertigung weitaus detaillierter. In Bezug auf die Spur „Tram“ beispielsweise wurde ursprünglich ein zunächst leichter Anstieg des Geräuschs auf eine bestimmte Lautstärke vermerkt, die dann laut Partitur für fast zwei Minuten durchgehend zu hören ist und zum Ende hin wieder abnimmt. Bei genauerem Anhören der Aufnahme wird jedoch deutlich, dass die Straßenbahn nur vereinzelt und nicht durchgehend zu hören ist. Geräusche des Grills wurden auf der ursprünglichen Partitur nur während der ersten Minute verzeichnet, tatsächlich sind diese allerdings auch im

18 Schafer, Die Ordnung der Klänge, S. 227-233. 
weiteren Zeitverlauf noch hörbar. Die Kindergeräusche wurden zunächst gar nicht notiert, was damit zusammenhängt, dass sie in der ursprünglichen Partitur einfach unter „Menschengemurmel“ fielen und nicht als solche kenntlich gemacht wurden. Selbiges gilt für "Schritte“, welche beim Hören im Nachhinein sehr markant in der Aufnahme erscheinen, aber im Gesamtklang während der Aufnahme ebenfalls im „Menschengemurmel“ untergegangen waren. Unter Rückbezug auf Schafer kann die Textur der akustischen Umgebung als Lo-Fi ausgewiesen werden.

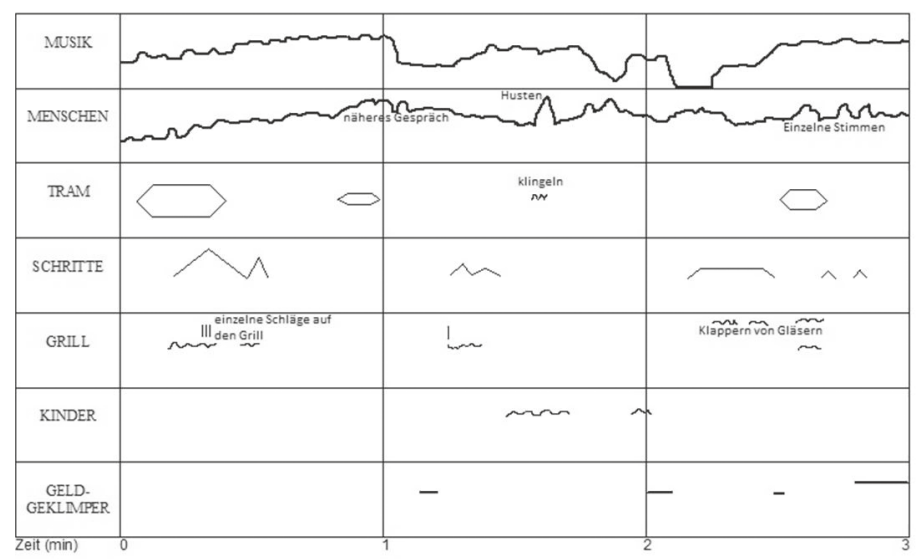

Abbildung 1: Korrigierte Hörpartitur zum Sub-Platz „Rapunzelturmweg“. Es werden die Parameter Dauer, Frequenz und Lautstärke abgebildet. Die Dauer verläuft an der x-Achse, die Frequenz wird bei Geräuschen als Fläche aufgezeichnet, und die Lautstärke bildet sich als gleichmäßiger (Linie) oder schwankender Verlauf (Welle) in ihrer jeweiligen Intensität ab.

\subsubsection{Nüsse (25. November 2014, $15.00 \mathrm{Uhr}$ )}

Am Sub-Platz „1000 Nüsse“ - dies ist der Name des entsprechenden Verkaufsstands - wurden ursprünglich sechs Klangschichten wahrgenommen: „Musik 1“, „Musik 2“, „Tram“, „Kasse“, „Standbesitzer“ und „Klopfen / Trommel“. Erstaunlicherweise war auf der Aufnahme nur eine Musikschicht zu hören, weshalb in der später korrigierten Partitur (siehe Abbildung 2) auch nur eine Musikspur vermerkt ist, welche sich durchgehend eher unauffällig im Hintergrund hält. Auch die Schicht „Klopfen / Trommel“ ist beim Anhören der Aufnahme nicht zu vernehmen. In Bezug auf das Straßenbahngeräusch zeigt sich das gleiche Phänomen wie bereits bei der oben beschriebenen bearbeiteten Partitur: Während zunächst praktisch durchgehende Tramgeräusche notiert wurden, fällt beim Hören im Nachhinein auf, dass es sich eigentlich um einzelne Geräuschquellen handelt. Im Prozess der Angleichung wurde die Spur „Kasse“ in „Geldgeklimper“ umbenannt. An dieser Stelle ist eine ähnliche Auffälligkeit zu beobachten wie bei „Tram“: Es sind wesentlich mehr vereinzelte Geräusche (vor allem in der zweiten Minute der Aufnahme) zu hören als zunächst notiert wurden. Interessant erscheint bei der Hörpartitur „1000 Nüsse“, dass im Gegensatz zu der Aufnahme „Rapunzelturmweg“ ursprünglich keinerlei menschliche Klangschichten aufgezeichnet wurden. Dies ist vor allem deshalb bemerkenswert, da die menschlichen Geräusche tatsächlich einen großen Teil der Geräuschkulisse ausmachen. Aus diesem Grund wurden für die Korrektur „Menschen“, „Schritte“ und „Kinder“ hinzugenommen, auch, 
um wieder eine Angleichung zur Partitur „Rapunzelturmweg“ anzustreben. Im Hinblick auf den Standbesitzer wurde im Gegensatz zur ursprünglichen Notierung zwischen Hintergrundgeräuschen und markanten Schlägen (dargestellt durch einzelne Striche) unterschieden. Auch hier handelt es sich also um eine Lo-Fi-Textur der akustischen Umgebung.

\begin{tabular}{|c|c|c|}
\hline Mussik & & 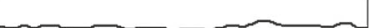 \\
\hline MENSCHEN & nate stimme & \\
\hline TRAM & $\Longrightarrow$ & $\stackrel{\text { Klingeli w }}{\rightleftharpoons}$ \\
\hline SCHRTITE & $\leadsto$ & 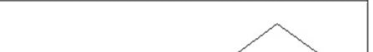 \\
\hline $\begin{array}{c}\text { STANDD } \\
\text { BESIIZRR }\end{array}$ & 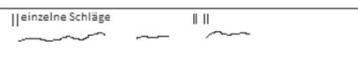 & 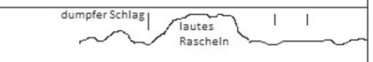 \\
\hline KINDER & & $\Omega \sqrt{ }$ \\
\hline GELD- & - & -- \\
\hline
\end{tabular}

Abbildung 2: Korrigierte Hörpartitur zum Sub-Platz „1000 Nüsse“. Es werden die Parameter Dauer, Frequenz und Lautstärke abgebildet. Die Dauer verläuft an der x-Achse, die Frequenz wird bei Geräuschen als Fläche aufgezeichnet, und die Lautstärke bildet sich als gleichmäßiger (Linie) oder schwankender Verlauf (Welle) in ihrer jeweiligen Intensität ab.

Es hat sich bezüglich beider Hörpartituren gezeigt, dass es erforderlich ist, im Nachhinein die jeweils dazugehörigen Soundscape-Aufnahmen genau zu analysieren. Wie an den beiden Beispielen zu sehen ist, gehen innerhalb der konkreten Situation viele Aspekte unter, bzw. Gehörtes wird in der Echtzeitsituation anders wahrgenommen oder aufgezeichnet, als es sich beim nachträglichen Analysieren darstellen lässt. Dies betrifft vor allem die technischen Klangschichten wie z. B. die Straßenbahngeräusche, welche in beiden ursprünglichen Hörpartituren als fast durchgehend dargestellt wurden, tatsächlich aber nur vereinzelt in der Aufnahme auftauchen. Auch die Zusammenfassung sämtlicher menschlicher Geräusche unter einer Klangschicht ist zwar nicht verkehrt, jedoch schwer zu notieren, da ein Husten beispielsweise ganz anders notiert werden sollte als Hintergrundgemurmel oder Schritte. Insgesamt ist es also sinnvoll, den Höreindruck der Momentaufnahme im Nachhinein noch einmal zu überprüfen und zu vergleichen, da die Differenzierung innerhalb der konkreten Situation doch recht schwierig erscheint.

Als Datenvermittlungsinstrument und Ergebnis der Soundscape-Aufnahmen wurde eine Klanglandkarte in Form einer Prezi-Präsentation erstellt, die interessierten Leserinnen und Lesern dieses Artikels einen virtuellen Rundgang über den Märchenweihnachtsmarkt mit einigen Hörproben ermöglicht. ${ }^{19}$ Hier sind auch die Lagepläne des Weihnachtsmarktes am Opernplatz, Friedrichsplatz und Königsplatz eingefügt. Diese zeigen den Aufbau der Buden und Fahrgeschäfte sowie die Straßennamen, die für den Weihnachtsmarkt gelten. Die Musikwissenschaftlerin Christa Brüstle hebt die identitätsstiftende Wirkung von Klangkarten hervor: „Klangkarten [...] fördern [...] die intersubjektive Erinnerung an Orte oder Begebenheiten oder eine kollektive Wahrnehmung bestimmter Vorgänge an

19 Siehe https://prezi.com/ow1rytni20qh/soundscape/ [7.2.2016]. 
einem Ort, haben also auch eine identitätsstiftende Wirkung, beispielsweise bezogen auf eine Stadt, Region oder einen bestimmten ,Treffpunkt ' [Hervorhebung im Original]. “20

Nähere Informationen über das Beschallungskonzept ermöglichte ein Expertengespräch mit Sebastian Schulze-von Hanxleden, dessen Firma Ton Direkt GmbH im Auftrag von Kassel Marketing GmbH seit etwa sieben Jahren die Zentralbeschallung des Weihnachtsmarktes übernimmt. Den Standbetreibern sei es laut Schulze-von Hanxleden zwar grundsätzlich möglich, eigene Musik „nicht zu laut“21 abzuspielen, der Großteil verzichte allerdings darauf. Kaufhäuser, angrenzende Läden und Filialen sind unabhängig von der kontrollierten Zentralbeschallung. Mit diesen erfolgt keine nähere Abstimmung. Die Musik, die zentral gespielt wird, soll qualitativ hochwertig und gleichzeitig frei von Ansprüchen seitens der Gesellschaft für musikalische Aufführungs- und mechanische Vervielfältigungsrechte (GEMA) sein. Eine weitere Vorgabe des Auftraggebers ist es, dass die Musik nicht im Vordergrund, sondern angenehm und unaufdringlich im Hintergrund zu hören sein soll. Dabei ist es wichtig, Musik mit einem hohen Bekanntheitsgrad auszuwählen, da so ein „Wohlfühlfaktor“ erreicht werden könne, wie beispielsweise beim wiederholten Sehen einer Lieblingsserie. Besondere Titelwünsche gebe es von Seiten der Kassel Marketing $\mathrm{GmbH}$ eher selten. Ziel der Musik sei es, dass die Leute möglichst lange auf dem Weihnachtsmarkt bleiben und sich wohlfühlen. Musikalische Experimente könnten Menschen vom Weihnachtsmarkt vertreiben und werden daher von der Stadt Kassel abgelehnt.

Die Titelliste umfasst ca. 100 Einträge, welche im Zufallsmodus wiedergegeben eine Spieldauer von ungefähr fünfeinhalb Stunden erreichen, bevor Wiederholungen entstehen. Vor dem ersten Advent wird eine andere Playlist mit Titeln abgespielt, die nicht eindeutig mit Weihnachten verbunden werden, beispielsweise die Brandenburgischen Konzerte von Johann Sebastian Bach (BWV 1046-1051). Da die Dynamik der einzelnen Musikstücke sehr unterschiedlich ist, werden diese stark komprimiert, um eine einheitliche Lautstärke zu erzielen. Durch den Marktmeister kann an den drei Hauptplätzen die absolute Lautstärke zentral reguliert werden.

\subsection{Erhebung mit Kurzfragebögen und themenzentrierten Interviews}

Zu der Beantwortung der Frage, inwieweit Musik auf dem Weihnachtsmarkt als Funktionelle Musik im Hintergrund zum Zweck der Verhaltenskonditionierung (siehe Kapitel 1.4) beiträgt, waren Befragungen hilfreich. Zunächst wurden mit Kurzfragebögen für Weihnachtsmarktbesucherinnen und -besucher überwiegend quantitative Angaben (z. B. durch Bewertungen mit den Schulnoten von 1 bis 6) erfasst. Außerdem gab es für die Befragten die Möglichkeit, eine Frage mit „keine Angabe“ zu bewerten, und für einige Fragen konnten selbst Zahlenwerte vergeben werden (z. B. „Ihr wievielter Weihnachtsmarktbesuch ist das in diesem Jahr?"). Der Bogen beinhaltete vier Fragekategorien:

20 Christa Brüstle, „Akustische Landaufnahme und Klangkartographie. Hörbare Welt und ihre Archive“, in: Kunsttexte.de 2/2013, http://edoc.hu-berlin.de/kunsttexte/2013-2/bruestle-christa-1/PDF/bruestle. pdf, 3.9.2015, S. 4.

21 Quelle dieses und der dazugehörigen Zitate ist die Transkription des Expertengesprächs vom 16.12.2014. 
1. Beurteilung des Weihnachtsmarkts mit Schulnoten (z. B. gastronomisches Angebot, Wetter, Größe, Weihnachtsstimmung),

2. Persönliche musikalische Präferenzen

3. Musik auf dem Weihnachtsmarkt (z. B. „Mögen Sie Weihnachten?“, oder „Mögen Sie Weihnachtsmusik?"),

4. Soziodemographische Angaben

Darüber hinaus wurde die Nachwirkung von Musik durch Auftreten von „Ohrwürmern“ abgefragt. Neben den bereits erwähnten Soundscapes und dem Expertengespräch wurden darüber hinaus themenzentrierte Interviews mit im Einzelhandel beschäftigten Personen geführt, die an ihren Ständen oder in ihren Geschäften dauerhaft Weihnachtsmusik ausgesetzt sind.

\section{Ergebnisse}

\subsection{Zu den Besucherinnen und Besuchern}

Die Studie erreichte $N=243$ Weihnachtsmarktbesucherinnen und -besucher, von denen $55,5 \%$ weiblich und 45,5\% männlich waren. Der Altersdurchschnitt lag bei 43,4 Jahren mit einer Spannweite von 14 bis 95 Jahren. Im multimodalen Ensemble der Angebote erfährt Musik eine nur befriedigende Schulnotenbewertung (3,0) (siehe Abbildung 3).

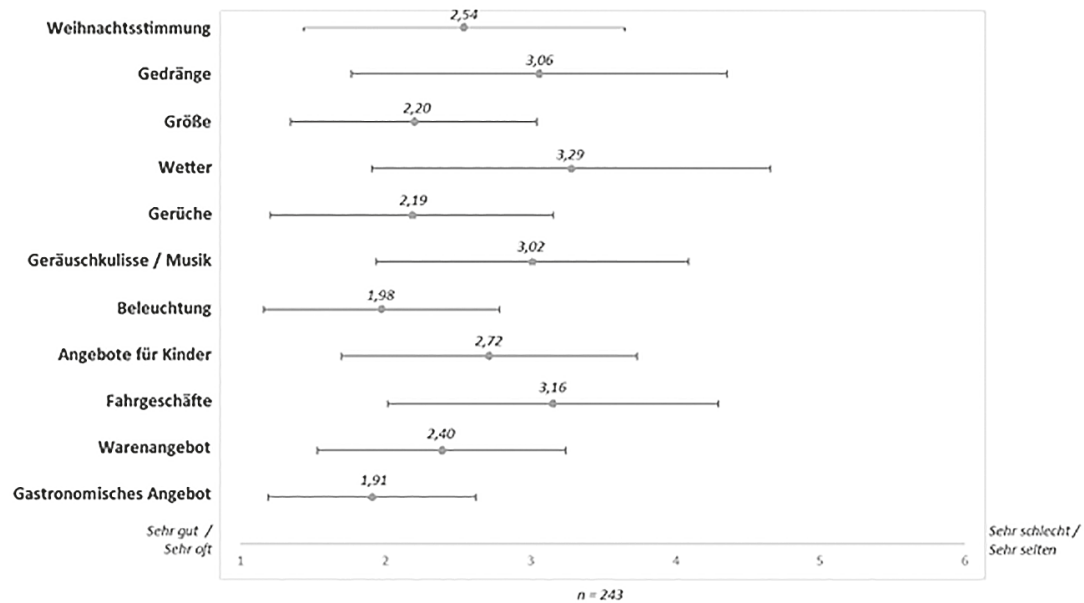

Abbildung 3: Beurteilung des Märchenweihnachtsmarkts. Mittelwerte (M) und Standardabweichungen (SD)

Dies liegt nicht etwa an ihrer Aufdringlichkeit, sondern vielmehr daran, dass Musik an vielen Stellen des Weihnachtsmarktes nicht oder nur andeutungsweise zu vernehmen war. Dies war bereits aus der Aufzeichnung von Soundscapes und Erstellung der Klanglandkarten hervorgegangen. Auch das Expertengespräch zeigte bereits, dass vom Auftraggeber möglichst unaufdringliche Musik beabsichtigt sei. Allerdings steht dies im Kontrast zur Meinung der Weihnachtsmarktbesucherinnen und -besucher, wo Weihnachtsmusik mit einer guten Durchschnittsnote $(2,5)$ zusammen mit Pop / Mainstream $(2,4)$ und SingerSongwriter / Liedermacher $(2,5)$ die vorderen Plätze unter den Musikpräferenzen belegt.

Um den Zusammenhang zwischen der Präferenz für Weihnachtsmusik und der Präferenz für ein Genre zu ermitteln, wurden explorative Pearson-Korrelationen (r) berechnet. 
Der zweiseitige Test hat ergeben, dass diejenigen, die im Alltag Schlager / Volksmusik, Klassik und Jazz präferieren, auch Weihnachtsmusik mögen (siehe Abbildung 4). Dagegen empfinden Techno/House/Elektro-Fans eine Abneigung gegen Weihnachtsmusik (siehe ebd.).

\begin{tabular}{|c|c|c|c|c|}
\hline Variable A & Variable B & $r$ & $\mathrm{p}$ & $N$ \\
\hline Gefallen Weihnachtsmusik & Gefallen Pop/Mainstream & 0,02 & 0,742 & 236 \\
\hline Gefallen Weihnachtsmusik & Gefallen Schlager/Volksmusik & $\mathbf{0 , 2 8} \mathbf{8}^{* *}$ & $\mathbf{0 , 0 0 0}$ & 237 \\
\hline Gefallen Weihnachtsmusik & Gefallen Klassik & $0,33^{* *}$ & $\mathbf{0 , 0 0 0}$ & 235 \\
\hline Gefallen Weihnachtsmusik & Gefallen Jazz & $0,24^{* *}$ & $\mathbf{0 , 0 0 0}$ & 231 \\
\hline Gefallen Weihnachtsmusik & Gefallen Singer-Songwriter/Liedermacher & 0,13 & 0,054 & 219 \\
\hline Gefallen Weihnachtsmusik & Gefallen Techno/House/Elektro & $-0,19^{* *}$ & 0,005 & 226 \\
\hline Gefallen Weihnachtsmusik & Gefallen Rock/Punk/Metal & $-0,11$ & 0,101 & 229 \\
\hline Gefallen Weihnachtsmusik & Gefallen Hip Hop & $-0,09$ & 0,171 & 228 \\
\hline Gefallen Weihnachtsmusik & Gefallen Sonstiges & $-0,10$ & 0,721 & 16 \\
\hline
\end{tabular}

Abbildung 4: Korrelationen

Eine explorative Gruppierung der Erhebungszeiträume früh - mittel - spät (Anfang Dezember - Mitte Dezember - kurz vor Weihnachten) und der Vergleich der dazugehörigen Bewertungen ergab keine Veränderung der Bewertung der Weihnachtslieder im Verlauf der Weihnachtszeit. Auch bei der Altersspanne lassen sich keine statistisch signifikanten Unterschiede in der Bewertung von Weihnachtsmusik feststellen.

„Last Christmas“ der Popgruppe Wham (1984) und „Jingle Bells“ (komponiert von James Lord Pierpont zwischen 1850 und 1857) wurden als verbreitetste „Ohrwürmer“ angegeben (siehe Abbildung 5). Grundsätzlich durchmischen sich in der Auflistung einschlägige Pop-Titel mit traditionellen Weihnachtsliedern. Nur letztere waren aufgrund der geforderten GEMA-Freiheit allerdings auch auf dem Weihnachtsmarkt zu hören. Es ist also zu vermuten, dass die „Ohrwürmer“ entweder durch andere akustisch-musikalische Kontexte oder durch allgemeine assoziative Trigger hervorgerufen wurden, ohne dass die Musik real erklungen sein muss. ${ }^{22}$ Grundsätzlich handelt es sich bei „Ohrwürmern“ um negativ oder positiv besetzte musikalische Gedächtnisinhalte, die sich teilweise auf sehr lange zurückliegende Lebensereignisse beziehen aber zugleich niederschwellig abrufen lassen. ${ }^{23}$

22 Hemming, „Zur Phänomenologie des,Ohrwurms““, S. 198.

23 Victoria Williamson, Sagar Jilka, Joshua Fry, Sebastian Finkel, Daniel Müllensiefen und Lauren Stewart, „How do ,earworms' start? Classifying the everyday circumstances of involuntary musical imagery“, in: Psychology of Music 40 (2012), S. 259-284. 


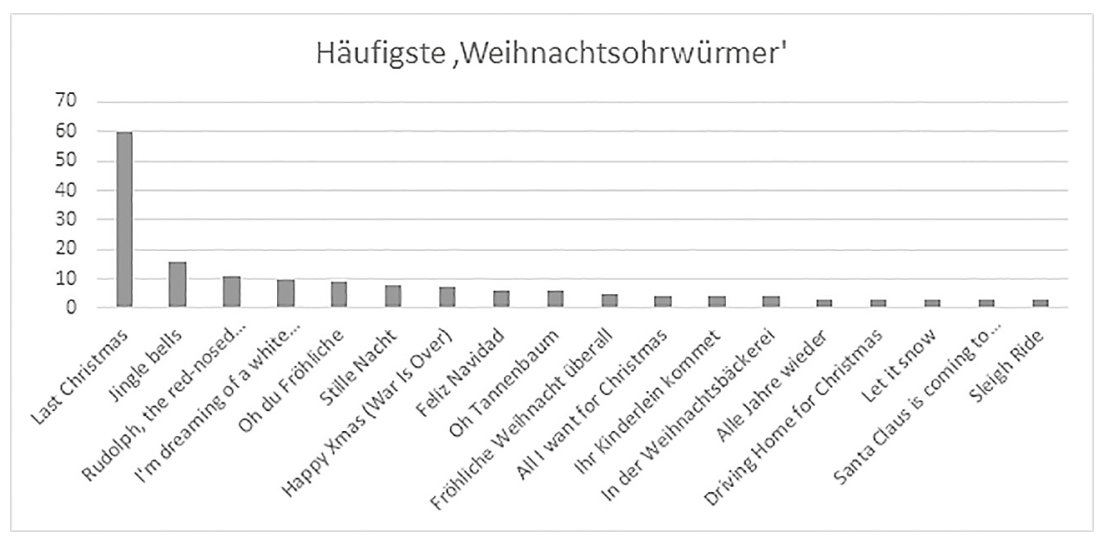

Abbildung 5: Weihnachtsohrwürmer

Ferner ist bekannt, dass „Ohrwürmer“ überwiegend bei präferierter Musik auftreten und auch als angenehm empfunden werden, wenn sie erst einmal da sind. ${ }^{24}$ Demgegenüber wurde das Auftreten der Weihnachtsohrwürmer wesentlich zurückhaltender mit einer schwach befriedigenden Durchschnittsnote von 3,4 bewertet. Den Gründen hierfür konnte nicht nachgegangen werden.

\subsection{Arbeitnehmerinnen und Arbeitnehmer}

Bei den Beschäftigten $(N=18)$ führt die ständige Wiederholung bestimmter Weihnachtslieder zusammen mit dem frühen Beginn des Weihnachtsmarktes (Ende November) unerwartet selten zu einer genervten Reaktion - „Weihnachtsfans“ sind davon kaum betroffen. Insgesamt gibt es gemischte Bewertungen (siehe Beispiele von positiven, neutralen und negativen Aussagen in Abbildung 6).

\begin{tabular}{|l|l|l|l|}
\hline Kürzel & Positiv & Neutral & Negativ \\
\hline AH & $\begin{array}{l}\text { „Also, ich mag Weihnachtsmusik } \\
\text { sehr gerne und freue mich da schon } \\
\text { das ganze Jahr drauf deswegen } \\
\text { empfinde ich das jetzt nicht so als } \\
\text { nervig oder so." (I01) }\end{array}$ & $\begin{array}{l}\text { Klar, gegen Ende hin wenn } \\
\text { man die Lieder alle schon 100 } \\
\text { mal gehört hat, dann denkt } \\
\text { man sich so ja jetzt könnte } \\
\text { auch mal wieder was anderes } \\
\text { kommen aber im Großen und } \\
\text { Ganzen bin ich da nicht so ge- } \\
\text { nervt von." (I01) }\end{array}$ & $\begin{array}{l}\text { "Na gut, wenn die Weihnachtsmusik } \\
\text { gespielt wird, ist es meistens nicht so } \\
\text { schön. Die ganzen Lieder kennt man } \\
\text { schon und hört die sich jedes Jahr } \\
\text { an. Die wiederholen sich auch noch } \\
\text { immer, das nervt. Ich und alle Mitar- } \\
\text { beiter sind immer froh, wenn Weih- } \\
\text { nachtsliederplaylist vorbei ist, und } \\
\text { wir ,normale Musik wieder hören } \\
\text { können." (I02) }\end{array}$ \\
\hline AM & & & \\
\hline
\end{tabular}

24 Hemming, „Zur Phänomenologie des ,Ohrwurms““, S. 201. 


\begin{tabular}{|c|c|c|c|}
\hline Kürzel & \begin{tabular}{|l|} 
Positiv \\
\end{tabular} & Neutral & Negativ \\
\hline AMM & $\begin{array}{l}\text { „In der Anfangsphase der Weih- } \\
\text { nachtszeit habe ich eine Vorfreude } \\
\text { auf die Weihnachtslieder. Diese } \\
\text { sind eine Abwechslung zu den ge- } \\
\text { wohnten Liedern. Außerdem freue } \\
\text { ich mich auf bekannte und neue } \\
\text { Weihnachtslieder." (I03) }\end{array}$ & & \\
\hline $\mathrm{CA}$ & $\begin{array}{l}\text { "Also allgemein ist die Musik eher } \\
\text { Nebensache in meinem Job ... } \\
\text { wobei man ihr natürlich quasi } \\
\text {,ausgesetzt" ist. Aber das finde ich } \\
\text { nicht störend, eher im Gegenteil, } \\
\text { sie macht eher gute Laune und alles } \\
\text { ein wenig angenehmer [...]“ (I04) }\end{array}$ & & \\
\hline $\mathrm{CU}$ & & $\begin{array}{l}\text { "Also [...] da kommt dann } \\
\text { teilweise noch so normale } \\
\text { Musik, dann kommen auch } \\
\text { viel so Weihnachtslieder, aber } \\
\text { nicht so unbedingt die klassi- } \\
\text { schen Weihnachtslieder, was } \\
\text { ich ganz gut finde, wenn ich } \\
\text { das den ganzen Tag hören müs- } \\
\text { ste würde ich wahrscheinlich } \\
\text { bekloppt werden. Aber halt } \\
\text { so, keine Ahnung, Backstreet } \\
\text { Boys Weihnachtslied oder Lady } \\
\text { Gaga Weihnachtslied kommt } \\
\text { dann ab und zu. Ja die Sache } \\
\text { ist, dass die sich ziemlich viel } \\
\text { wiederholen und dann schnell } \\
\text { nervig werden für dich wenn } \\
\text { du da arbeitest. Für die Kun- } \\
\text { den - die merken das wahr- } \\
\text { scheinlich nicht. Naja, es geht, } \\
\text { man hält es aus, es kann einem } \\
\text { aber schon auf die Nerven ge- } \\
\text { hen." (I05) }\end{array}$ & \\
\hline $\mathrm{FH}$ & & $\begin{array}{l}\text { "Naja, also anfangs ist es relativ } \\
\text { gemütlich, so Musik zu hören, } \\
\text { aber am Ende muss ich sagen } \\
\text { wird's dann doch eher nervig; } \\
\text { vor allem weil man auch im } \\
\text { Radio von allen Seiten damit } \\
\text { beschallt wird und dann ist es } \\
\text { irgendwann auch genug." (I06) }\end{array}$ & \\
\hline $\mathrm{JB}$ & & $\begin{array}{l}\text { "Ich meine, dass bei uns kei- } \\
\text { ne Weihnachtsmusik gespielt } \\
\text { wird, zumindest habe ich bis } \\
\text { jetzt noch keine wahrgenom- } \\
\text { men. Ich finde das aber auch } \\
\text { nicht schlimm, weil an anderen } \\
\text { Stellen genug Weihnachtsmu- } \\
\text { sik läuft." (I07) }\end{array}$ & \\
\hline JS & & $\begin{array}{l}\text { "Die ersten drei, vier Tage krie- } \\
\text { gen wir das richtig mit und } \\
\text { dann geht das hier rein und da } \\
\text { raus." (I08) }\end{array}$ & \\
\hline
\end{tabular}




\begin{tabular}{|l|l|l|l|}
\hline Kürzel & Positiv & Neutral & Negativ \\
\hline KG & & & „Also,...wenn ich ganz ehrlich bin, \\
& & & $\begin{array}{l}\text { ich bin ja jetzt net mehr der Jün- } \\
\text { ste, aber, ... man brauch's halt nicht } \\
\text { unbedingt, aber es wird halt einfach } \\
\text { von der Firma gefordert, dass man die } \\
\text { Musik anmacht.“ (I09) }\end{array}$ \\
\hline
\end{tabular}

Abbildung 6: Aussagen von ausgewählten Beschäftigten $(n=9)$

Grundsätzlich haben lediglich 28 \% der Beschäftigten einen Einfluss auf die Musikauswahl in ihrer unmittelbaren Arbeitsumgebung. Die Hälfte der Beschäftigten hat einen Einfluss auf die Musiklautstärke. Etwa zwei Drittel der Beschäftigten berichten davon, dass die Musik an ihrem Arbeitsplatz als „Ohrwurm“ nachwirkt. Jenseits der Weihnachtszeit lässt sich festhalten, dass sich Musik am Arbeitsplatz nur bei gut einem Drittel der Befragten (36\%) positiv auf die Zufriedenheit der Angestellten auswirkt. Kommt es hier zu „Ohrwürmern“, empfindet dies nur die Hälfte der Befragten als unangenehm.

Resultat einer qualitativen Inhaltsanalyse der Interviews mit offenen Kategorien ${ }^{25}$ ist die Herausbildung der Kategorie „Habituierung“. Weitgehend unabhängig von den zuvor erwähnten positiven, neutralen oder negativen Grundeinstellungen gegenüber Weihnachtsmusik lässt sich sehr deutlich beobachten, dass bei den Beschäftigten Gewöhnungseffekte eintreten, die dazu führen, dass die Weihnachtsmusik kaum noch bewusst wahrgenommen wird. Dies wird durch die Zitate in Abbildung 7 veranschaulicht.

\begin{tabular}{l}
\hline „Hierzu kann ich sagen, dass ich insbesondere die Weihnachtsmusik, die während der Adventszeit in \\
unserem Laden läuft, schon gar nicht mehr wirklich höre. Ich nehme die Musik nur noch unterbewusst \\
wahr, das verstärkt sich besonders hin zur Schlussphase der Weihnachtszeit.“ (I14) \\
\hline „Man nimmt es, wenn man beschäftigt ist, auch nicht unbedingt wahr.“ (I15) \\
\hline „Ich bemerke die Musik selbst eigentlich kaum. Sie ist sehr hintergründig.“ (I15) \\
\hline „Am Schluss nervt es (fast) gar nicht mehr, weil ich selbst in Weihnachtsstimmung bin.“ (I17) \\
\hline
\end{tabular}

Abbildung 7: Habituierung

Gleichwohl würde es den Beschäftigten möglicherweise entgegenkommen, wenn auch während der Weihnachtszeit eine Mischung von „normaler“ Musik und Weihnachtsmusik gespielt würde. Dies könnte sich zuletzt auch positiv auf die Kundinnen und Kunden auswirken.

\section{Resümee}

Abschließend bleibt festzuhalten, dass vor allem das vermutete Ausmaß und die vermutete Intensität der Weihnachtsmusik in einem Missverhältnis zur Realität stehen. Die beteiligten Forscherinnen und Forscher waren fast ein wenig ernüchtert darüber, wie wenig Weihnachtsmusik de facto zu hören war. Dadurch kann Weihnachtsmusik als Funktionelle Musik auf dem Kasseler Märchenweihnachtsmarkt substantiell kaum zu einer besseren Atmosphäre beitragen und keine verlässliche Grundlage der Überprüfung des musical „fit“ sein. Ähnliches belegten auch individuelle Besuche mit gesteigerter Aufmerksamkeit

25 Vgl. hierzu Jan Hemming, „Empirische Forschung“, in: Methoden der Erforschung populärer Musik, Wiesbaden 2016, S. 256-259. 
von Weihnachtsmärkten in Erfurt und Chemnitz. Offenbar hat sich ein akustisches Umweltbewusstsein auf breiter Front durchgesetzt, so dass von einer Reizüberflutung mit Weihnachtsmusik keine Rede mehr sein kann. Dies spiegelt sich in Vorgaben seitens der Auftraggeber, dass die Musik auf keinen Fall aufdringlich oder experimentell sein dürfe. Eine (stärkere) Mitbestimmung über die Qualität und Intensität der Musikauswahl seitens der Menschen, die sich auf dem Weihnachtsmarkt aufhalten, könnte das Funktionelle der Weihnachtsmusik optimieren anstatt auf Fremdbestimmung zu setzen. Zudem kann durch eine (stärkere) Mitbestimmung die Wahrnehmung und Wirkung von Weihnachtsmusik auf beide Gruppen (Beschäftigte und Besucher) besser abgestimmt werden. Eine wissenschaftliche Überprüfung oder öffentliche Diskussion der Wahrnehmung und Wirkung von Weihnachtsmusik im öffentlichen Raum findet kaum statt - ganz im Gegensatz etwa zur Weihnachtsbeleuchtung, über die in Kassels Presse seit Jahren heftig gestritten wird. Ist es ein Zufall, dass die Beleuchtung zusammen mit dem gastronomischen Angebot (siehe Abbildung 3) die beste Bewertung bekommt? Wenn sich an einer größeren Stichprobe andererseits bestätigen ließe, dass die Beschäftigten mehrheitlich nicht grundsätzlich unter Beschallung mit Weihnachtsmusik leiden oder diese durch Habituierungseffekte zu ignorieren lernen, stünde einer Ausweitung der Weihnachtsbeschallung grundsätzlich nichts im Wege. Drei Jahrzehnte nach Helga de la Mottes „Musik für alle Tage: Ein ästhetischer Gegenentwurf “26 könnte dies also der Moment sein, wo aus musikpsychologischer Sicht empfohlen wird, die Lautstärke der Hintergrundbeschallung des Kasseler Weihnachtsmarkts zu erhöhen - wohlgemerkt als Reaktion auf die Befragung seiner Besucher. Ganz wohl fühlen sich die Autoren dabei allerdings nicht. 